\title{
Evaluation of prescribing patterns in a German network of CAM physicians for the treatment of patients with hypertension: a prospective observational study
}

\author{
Elke Jeschke ${ }^{1}$, Thomas Ostermann*2, Horst C Vollmar ${ }^{3,4}$, Matthias Kröz ${ }^{1}$, \\ Angelina Bockelbrink ${ }^{5}$, Claudia M Witt ${ }^{5}$, Stefan N Willich ${ }^{5}$ and \\ Harald Matthes ${ }^{1}$
}

\begin{abstract}
Address: ${ }^{1}$ Havelhoehe Research Institute, Kladower Damm 221, 14089 Berlin, Germany, ${ }^{2}$ Chair for Theory of Medicine, Integrative and Anthroposophic Medicine, University of Witten/Herdecke, Gerhard-Kienle-Weg 4, 58313 Herdecke, Germany, ${ }^{3}$ Institute for General Practice and Family Medicine, University of Witten/Herdecke, Alfred-Herrhausen-Str 50, 58448 Witten, Germany, ${ }^{4}$ Fraunhofer Institute for Systems and Innovation Transfer (ISI), Breslauer Str 48, 76139 Karlsruhe, Germany and ${ }^{5}$ Institute for Social Medicine, Epidemiology and Health Economics, Charité University Medical Centre, 10117 Berlin, Germany

Email: Elke Jeschke - ejeschke@ havelhoehe.de; Thomas Ostermann* - thomaso@uni-wh.de; Horst C Vollmar - horst.vollmar@isi.fraunhofer.de; Matthias Kröz - mkroez@ havelhoehe.de; Angelina Bockelbrink - angelina.bockelbrink@charite.de; Claudia M Witt - claudia.witt@charite.de; Stefan N Willich - stefan.willich@charite.de; Harald Matthes - hmatthes@havelhoehe.de

* Corresponding author
\end{abstract}

Published: 10 December 2009

BMC Family Practice 2009, 10:78 doi:10.1186/147I-2296-10-78
Received: 14 July 2009

Accepted: 10 December 2009

This article is available from: http://www.biomedcentral.com/I47I-2296// 0/78

(C) 2009 Jeschke et al; licensee BioMed Central Ltd.

This is an Open Access article distributed under the terms of the Creative Commons Attribution License (http://creativecommons.org/licenses/by/2.0), which permits unrestricted use, distribution, and reproduction in any medium, provided the original work is properly cited.

\begin{abstract}
Background: The management of hypertension is a key challenge in modern health systems. This study aimed to investigate hypertension treatment strategies among physicians specialized in complementary and alternative medicine (CAM) in Germany by analysing prescribing patterns and comparing these to the current treatment guidelines issued by the German Hypertension Society.

Methods: In this prospective, multicentre observational study, which included 25 primary care physicians specialized in CAM treatment, prescriptions and diagnoses were analysed for each consecutive hypertensive patient using routine electronic data. Data analysis was performed using univariate statistical tests (Chi square test, Cochran-Armitage trend test). Multiple logistic regression was used to determine factors associated with antihypertensive medication.

Results: In the year 2005, I320 patients with 3278 prescriptions were included (mean age $=64.2$ years (SD $=14.5), 63.5 \%$ women). Most patients were treated with conventional antihypertensive monotherapies $(n=838,63.5 \%)$. Beta-blockers were the most commonly prescribed monotherapy (30.7\%), followed by ACE inhibitors (24.0\%). Combination treatment usually consisted of two antihypertensive drugs administered either as separate agents or as a coformulation. The most common combination was a diuretic plus an ACE inhibitor (3I.2\% of dual therapies). Patient gender, age, and comorbidities significantly influenced which treatment was prescribed. 187 patients (14.2\%) received one or more CAM remedies, most of which were administered in addition to classic monotherapies $(n=104)$. Men $(O R=0.66 ; 95 \% \mathrm{Cl}: 0.54-0.80)$ and patients with diabetes (OR $=0.55 ; 95 \% \mathrm{Cl}: 0.42-0.0 .73)$, hypercholesterolaemia $(\mathrm{OR}=0.59 ; 95 \% \mathrm{Cl}: 0.47-0.75)$, obesity ( $\mathrm{OR}=0.74 ; 95 \% \mathrm{Cl}: 0.57-0.97)$, stroke $(\mathrm{OR}=0.54 ; 95 \% \mathrm{Cl}: 0.40-0.74)$, or prior myocardial infarction $(O R=0.37 ; 95 \% \mathrm{Cl}: 0.17-0.8 \mathrm{I})$ were less likely to receive CAM treatment.
\end{abstract}


Conclusions: The large majority of antihypertensive treatments prescribed by CAM physicians in the present study complied with the current German Hypertension Society treatment guidelines. Deviations from the guidelines were observed in one of every seven patients receiving some form of CAM treatment.

\section{Background}

The management of hypertension is a key challenge in modern health systems. One of the most frequent chronic conditions and the most common treatable risk factor for cardiovascular disease, hypertension has been estimated to account for $6 \%$ of deaths worldwide [1] and for $26 \%$ of total mortality in Germany [2]. Hypertension was observed in $27 \%$ of women and $30 \%$ of men in Germany in a study by Thamm [3], and more recent studies have reported a prevalence of more than $50 \%$ in the general population [4].

Hypertension is the most common diagnosis made by general practitioners (GPs) in Germany [5], and antihypertensive agents are the second most frequently prescribed class of drugs, accounting for $15.4 \%$ of total drug expenditure [6]. Nevertheless, Germany is lagging behind internationally in areas such as hypertension awareness, treatment, and control [7]. Indeed, surveys have indicated that $41 \%$ of patients with known hypertension in this country are prescribed an inadequate dose of antihypertensive drugs or take these at dosing intervals that deviate from standard treatment recommendations [8]. Some experts have also criticized the use of newer and more expensive drugs, which in many cases may not be any more effective than older and less expensive treatments, such as diuretics [9].

The development, dissemination, and implementation of hypertension treatment guidelines are a key strategy for improving the care of hypertensive patients. Although several such guidelines have been published in Germany over the past decade, the most widely disseminated of these [10] were developed by the German Hypertension Society [11]. Moreover, recommendations have also been published for hypertensive patients themselves to ensure that they are kept abreast of the latest advances in treatment $[12,13]$.

Some studies have indicated that complementary and alternative remedies from the areas of phytotherapy, homoeopathy, or anthroposophy have potential in the treatment of hypertension [14]. Whereas phytotherapy involves the use of undiluted plant extracts, homoeopathy relies on minerals, botanical substances, and other sources in diluted form. Anthroposophic remedies include preparations of botanical, mineral, or zoological origin, as well as chemical substances that are either undi- luted or based on the homoeopathic principle of high dilution [15]. Three remedies in particular have shown some effects in the treatment of mild to moderate hypertension: crataegus [16,17], ginger [18], and Cardiodoron ${ }^{\circledR}$ - a mixture of extracts from Hyoscyamus niger (Henbane) and the blossoms of Primula veris (Cowslip) and Onopordum acanthium (Scotch thistle) [19]. According to a systematic review by Ernst et al. [20], however, the effect sizes of complementary and alternative remedies in the treatment of hypertension are modest. To date, such remedies have not been included in evidence-based guidelines or patient recommendations for hypertension.

Nevertheless, the acceptance of complementary and alternative medicine (CAM) has grown over the past 20 years among physicians and patients alike. Patients with chronic diseases, such as hypertension, are increasingly seeking CAM treatment [21-23]. As a result, a growing number of physicians are being asked by their patients for advice on CAM or for referrals to its practitioners [24].

An important barrier to guideline adherence is the difficulty faced by physicians in reconciling patient preferences with guideline recommendations [25]. According to studies in a variety of primary care settings, patient expectations and preferences can influence the health care provided to them [26-28]. This issue may be particularly salient in the setting of CAM, as patients seeking treatment from physicians specialized in this field are likely to expect to receive some form of alternative therapy. The present study thus aims (a) to investigate adherence among primarycare physicians specialized in CAM to the hypertension treatment guidelines issued by the German Hypertension Society in 2005 and (b) to examine the use of complementary remedies among patients treated by these physicians by analysing prescribing patterns.

\section{Methods}

In total, 25 primary care physicians in Germany participated in this prospective, multicentre observational study. All of them were members of the EvaMed Network, which aims to evaluate CAM remedies in usual care with regard to prescribing patterns, efficacy, and safety [29-31]. Physicians were recruited through the German National Association of Anthroposophic Physicians (Gesellschaft Antroposophischer Ärzte in Deutschland; GAÄD). A total of 362 physicians were contacted and informed about the EvaMed Network by standard mail and, in the event of 
non-response, four weeks later by telephone. For a physician to be eligible to participate in the study, his or her medical practice had to meet a number of technical requirements, including the presence of a special computerized patient documentation system (DocExpert, DocConcept, TurboMed, Duria, AdamedPlus, Medistar), a local area network (LAN) connection, and Microsoft Windows and Internet Explorer (i.e. as client software). A total of 38 physicians $(10.5 \%)$ fulfilled the technical requirements, gave informed consent, and agreed to participate in the EvaMed network. Of these physicians, 13 specialized in paediatrics and dermatology were excluded from the study. Each of the remaining 25 physicians had practised for at least five years in primary care in addition to completing training in anthroposophic medicine.

The present study is based on secondary data provided by physicians. As such, the recommendations for good practice in secondary data analysis (e.g. anonymization of data on prescriptions and diagnoses) developed by the German Working Group on the Collection and Use of Secondary Data [32] were applied in full. In addition, the study was approved by the responsible data security official.

Data were included in the study if patients were at least 16 years old, had been diagnosed with hypertension, and had received pharmacological treatment for hypertension at least once during the study period (January-December 2005).

During the study, physicians continued to follow their routine documentation procedures, recording diagnoses and all prescriptions for each consecutive patient using their existing, computerized patient documentation system. These data were exported to the QuaDoSta postgreSQL database hosted in each practice [33]. Physicians used a browser-based interface to match individual diagnoses with the corresponding drugs or remedies that had been prescribed. Diagnoses were coded according to the 10th revision of the International Classification of Diseases (ICD-10). Prescribed drugs were documented using the German National Drug Code.

Study investigators identified all drugs and remedies prescribed for hypertension (i.e. ICD-10: I10 - I15). Each substance was classified using the Anatomical Therapeutic Chemical Index, and hypertensive drugs were clustered into classic antihypertensives (i.e. calcium channel blockers (CCBs), diuretics, beta-blockers, angiotensin converting enzyme (ACE) inhibitors, angiotensin II receptor antagonists, alpha-1 blockers, and antiadrenergic agents), and combination treatments (e.g. diuretics and betablockers, either as fixed-dose coformulations or as separate agents).
All statistical analyses were performed using SPSS 16.0 for Windows. Mean and standard deviations (SD) were calculated for continuous, normally distributed data. In cases where data were not normally distributed, medians and interquartile ranges (IQR) were reported. Subgroup analyses of prescribing rates were performed for patient age (under 40 years, $40-59$ years, 60-79 years, 80 years and older), gender, and co-morbidities (e.g. diabetes mellitus, renal insufficiency, hypercholesterolaemia, coronary heart disease (CHD), post myocardial infarction, heart failure, stroke, obesity, asthma/chronic obstructive pulmonary disease (COPD)). The two-tailed Chi square test was used to analyse differences in prescription rates, and the Cochran-Armitage test was used as a measure of trend. A $P$ value of less than 0.05 was regarded as indicating a statistically significant difference. Adjusted odds ratios (OR) and $95 \%$ confidence intervals (CI) were calculated using multiple logistic regression to determine factors associated with different hypertensive medications (CCBs, diuretics, beta-blockers, ACE inhibitors, angiotensin II antagonists, or CAM remedies).

\section{Results \\ Physicians}

Of the 25 participating physicians, 21 were GPs ( $84 \%$ ) and 4 were internists (16\%). The physicians did not differ significantly from the overall population of physicians certified in anthroposophy in Germany $(n=362)$ in terms of age $($ mean $=49.4 ; \mathrm{SD}=6.3$ years vs. mean $=47.5 ; \mathrm{SD}=$ 6.1 years; $P=0.709)$ or gender $(60.0 \%$ vs. $62.2 \%$ men; $P$ $=0.917)$, and were only slightly younger and consisted of a similar percentage of women compared to all officebased physicians in Germany (mean 52.0 years; $61.2 \%$ men) [34].

\section{Patients and prescriptions}

During the study period, 8978 primary care patients (60.5\% women) were treated by the participating physicians. Of the 3672 patients who were older than 16 years (40.9\%), a total of 1320 (63.5\% women) met the inclusion criteria $(14.7 \%)$. The mean age of the included patients was 64.2 years $(S D=14.5)$. A total of 1613 comorbidities were recorded (median: 1 ; IQR $[0,2])$. The highest comorbidity rates were seen in patients aged 60 to 79 years ( 1.20 in women and 1.61 in men) and patients aged 80 years and older (1.70 in women and 1.82 in men). In total, $32.4 \%$ of all patients had one, $19.8 \%$ had two, and $12.8 \%$ had three or more comorbidities. Thirtyfive percent of patients $(n=462)$ had no reported comorbidities. Table 1 provides a detailed overview of comorbidities in participating patients.

During the study period, 1320 patients received a total of 3278 prescriptions (median prescriptions per patient and year: 1.5 ; IQR $[1.0,3.0])$. Most patients $(\mathrm{n}=838 ; 63.5 \%)$ 
Table I: Sample of hypertensive patients subdivided according to comorbidities, age group, and gender

\begin{tabular}{|c|c|c|c|c|c|c|c|c|c|c|c|}
\hline \multirow{3}{*}{$\begin{array}{l}\text { Age } \\
\text { group } \\
\text { [years] }\end{array}$} & \multirow{3}{*}{$\begin{array}{c}\text { Total } \\
\\
\mathbf{N}\end{array}$} & \multirow{3}{*}{$\begin{array}{c}\begin{array}{c}\text { Without } \\
\text { comorbidities }\end{array} \\
{[n(\%)]}\end{array}$} & \multicolumn{9}{|c|}{ Comorbidities I } \\
\hline & & & \multirow{2}{*}{$\begin{array}{c}\begin{array}{c}\text { Diabetes } \\
\text { mellitus }\end{array} \\
{[n(\%)]}\end{array}$} & \multirow{2}{*}{$\begin{array}{c}\begin{array}{c}\text { Renal } \\
\text { insufficiency }\end{array} \\
{[n(\%)]}\end{array}$} & \multirow{2}{*}{$\begin{array}{c}\begin{array}{l}\text { Hypercholest } \\
\text { erolaemia }\end{array} \\
{[n(\%)]}\end{array}$} & \multirow{2}{*}{$\begin{array}{c}\begin{array}{c}\text { Coronary } \\
\text { heart } \\
\text { disease }\end{array} \\
{[n(\%)]}\end{array}$} & \multirow{2}{*}{$\begin{array}{c}\begin{array}{c}\text { Post } \\
\text { myocardial } \\
\text { infarcttion }\end{array} \\
{[n(\%)]}\end{array}$} & \multirow{2}{*}{$\begin{array}{c}\begin{array}{c}\text { Heart } \\
\text { failure }\end{array} \\
\text { [n (\%)] }\end{array}$} & \multirow{2}{*}{$\begin{array}{l}\text { Stroke } \\
{[n(\%)]}\end{array}$} & \multirow{2}{*}{$\begin{array}{l}\text { Obesity } \\
\text { [n (\%)] }\end{array}$} & \multirow{2}{*}{$\begin{array}{c}\begin{array}{c}\text { Asthma/ } \\
\text { COPD }\end{array} \\
\text { [n (\%)] }\end{array}$} \\
\hline & & & & & & & & & & & \\
\hline Under 40 & 23 & 14 (60.9) & I (4.3) & $2(8.7)$ & $2(8.7)$ & I (4.3) & $0(0.0)$ & $0(0.0)$ & I (4.3) & $4(17.4)$ & I (4.3) \\
\hline Female & 27 & $17(63.0)$ & I (3.7) & $2(7.4)$ & $2(7.4)$ & $2(7.4)$ & $0(0.0)$ & $0(0.0)$ & $0(0.0)$ & $6(22.2)$ & $4(14.8)$ \\
\hline \multicolumn{12}{|l|}{ Male } \\
\hline \multicolumn{12}{|l|}{$40-59$} \\
\hline Female & 248 & $124(50.0)$ & $23(9.3)$ & $8(3.2)$ & $62(25.0)$ & $12(4.8)$ & $0(0.0)$ & $2(0.8)$ & $9(3.6)$ & $62(25.0)$ & $10(14.9)$ \\
\hline Male & 164 & $63(38.4)$ & $19(11.6)$ & $9(5.5)$ & $69(42.1)$ & $18(11.0)$ & $5(3.0)$ & I (0.6) & $5(3.0)$ & $50(30.5)$ & $9(19.6)$ \\
\hline \multicolumn{12}{|l|}{$60-79$} \\
\hline Female & 406 & $\mid 28$ (3|.5) & $82(20.2)$ & $17(4.2)$ & $167(4||)$. & $84(20.7)$ & $4(1.0)$ & 15 (3.7) & $47(11.6)$ & 71 (I7.5) & $37(55.2)$ \\
\hline Male & 247 & 7I (28.7) & $64(25.9)$ & $18(7.3)$ & $96(38.9)$ & $68(27.5)$ & $17(6.9)$ & $10(4.0)$ & $24(9.7)$ & $30(12,1)$ & $24(52.2)$ \\
\hline \multicolumn{12}{|l|}{$\begin{array}{l}80 \text { or } \\
\text { older }\end{array}$} \\
\hline Female & 161 & $39(24.2)$ & $46(28.6)$ & $18(11.2)$ & 35 (21.7) & $56(34.8)$ & $6(3.7)$ & $4(2.5)$ & $5 \mathrm{I}(3 \mathrm{I} .7)$ & $19(11.8)$ & $19(28,4)$ \\
\hline Male & 44 & $6(13.6)$ & $10(22.7)$ & $7(\mid 5.9)$ & $13(29.5)$ & $17(38.6)$ & $3(6.8)$ & $2(4.5)$ & $17(38.6)$ & $5(11.4)$ & $9(19,6)$ \\
\hline Total & 1320 & $462(35.0)$ & $\begin{array}{c}246 \\
(16.6)\end{array}$ & $81(6.1)$ & $446(33.8)$ & $\begin{array}{c}258 \\
(19.5)\end{array}$ & $35(2.7)$ & $34(2.6)$ & $\begin{array}{c}154 \\
\text { (II.7) }\end{array}$ & $\begin{array}{c}247 \\
(18.7)\end{array}$ & I I 3 (8.6) \\
\hline
\end{tabular}

I Double entries possible

received classic antihypertensive monotherapy, which accounted for 1705 prescriptions $(52.0 \%$ of all prescriptions). A total of 482 patients (36.5\%) received combination treatment with two antihypertensive drugs, which accounted for 845 prescriptions $(25.8 \%$ of all prescriptions). Triple combinations were prescribed for 69 patients $(5.2 \%)$, accounting for 104 prescriptions $(3.2 \%$ of all prescriptions).

\section{Pharmacological therapy}

A total of 187 patients (14.2\%) were prescribed CAM remedies, accounting for 624 prescriptions $(19.1 \%$ of all prescriptions). Of these prescriptions, $155(4.7 \%$ of all prescriptions) were for CAM remedies administered as an adjunct to conventional therapy. Most frequently, this involved CAM prescribed in addition to classic monotherapy $(n=104)$, especially among women $(n=79)$. In total, 97 patients $(7.3 \%)$ received antihypertensive CAM treatment exclusively (i.e. without any form of conventional therapy).

The most commonly prescribed agents were the betablocker metoprolol (9.5\%), the ACE inhibitor enalapril (5.8\%), the beta-blocker bisoprolol (4.4\%), the CCB amlodipine (3.9\%), the ACE inhibitor ramipril (3.3\%), and the diuretic thiazide (3.0\%). The most commonly prescribed CAM remedies were Cardiodoron ${ }^{\circledast}(4.0 \%)$ and various aurum preparations $(3.8 \%$; e.g. Aurum/Bella- donna comp ${ }^{\circledast}$, Aurum metallicum praeparatum ${ }^{\circledast}$, Aurum naturale D10/Punus spinosa, Summitates D5 ${ }^{\varpi}$ ).

For monotherapy, the most frequently prescribed agents were beta-blockers $(30.7 \%)$, followed by ACE inhibitors $(24.0 \%)$, CCBs (16.7\%), and diuretics (15.7\%). No differences could be seen in the prescription rates between the genders for specific monotherapies, such as beta-blockers $(29.0 \%$ in men and $31.7 \%$ in women; $P=0.525)$ and angiotensin II receptor antagonists $(14.6 \%$ in men and $10.1 \%$ in women; $P=0.332$ ). The overall proportion of monotherapies also did not differ between men and women $(P=0.079$; see table 2 for details). However, there were differences in the prescription rates for monotherapeutic agents between the various age groups. Beta-blockers, for example, were prescribed most frequently in patients aged less than 60 years, with $47.6 \%$ of patients aged between 20 and 39 years and $39.5 \%$ of patients aged between 40 and 59 years receiving these agents. In contrast, beta-blocker monotherapy was prescribed to only $28.1 \%$ of patients aged 60 to 79 years and $21.9 \%$ of patients aged 80 years and older $(P$ for trend $<0.001)$. Among patients aged 80 years and older, diuretics $(24.3 \%)$, ACE inhibitors (24.3\%), CCBs $(23.7 \%)$, and beta-blockers $(21.9 \%)$ were prescribed with almost equal frequency. Compared to the average, however, these patients received a significantly higher proportion of CCBs and diuretics. Only every eighth patient (12.9\%) 
Table 2: Prescription of monotherapies according to age group and gender

\begin{tabular}{|c|c|c|c|c|c|c|c|}
\hline \multirow[t]{2}{*}{ Antihypertensives } & \multirow[t]{2}{*}{ Total } & \multicolumn{2}{|c|}{ Gender } & \multicolumn{4}{|c|}{ Age group [years] } \\
\hline & & Male & Female & Under 40 & 40-59 & $60-79$ & 80 or older \\
\hline & [N (\%)] & [n (\%)] & [n (\%)] & [n (\%)] & [n (\%)] & [n (\%)] & [n (\%)] \\
\hline $\mathrm{CCBs} \mathrm{s}^{\prime}$ & $285(16.7)$ & $101(16.2)$ & $184(17.0)$ & $12(19.0)$ & $47(9.1)$ & $148(18.5)$ & $78(23.7)$ \\
\hline - with CAM² & 9 & 2 & 7 & - & 1 & 6 & 2 \\
\hline Diuretics & 267 (15.7) & $88(14.1)$ & $179(16.6)$ & $9(14.3)$ & 71 (13.7) & $107(13.4)$ & $80(24.3)$ \\
\hline - with CAM & 28 & 8 & 20 & 1 & 16 & 9 & 2 \\
\hline Beta-blockers & $523(30.7)$ & $|8|(29.0)$ & $342(31.7)$ & $23(36.5)$ & $204(39.5)$ & $224(28.1)$ & $72(21.9)$ \\
\hline - with CAM & 27 & 7 & 20 & 1 & 8 & 15 & 3 \\
\hline ACE inhibitors & $409(24.0)$ & $158(25.3)$ & $25 I(23.2)$ & $17(27.0)$ & $106(20.5)$ & $206(25.9)$ & $80(24.3)$ \\
\hline - with CAM & 23 & 3 & 20 & 1 & 6 & 10 & 6 \\
\hline Angiotensin II antagonists & 200 (1I.7) & $91(14.6)$ & $109(10.1)$ & $\mathrm{I}(1.6)$ & $79(15.3)$ & $104(13.1)$ & $16(4.9)$ \\
\hline - with CAM & 17 & 5 & 12 & - & 7 & 8 & 2 \\
\hline Alpha-I blockers & $4(0.2)$ & $2(0.3)$ & $2(0.2)$ & - & $3(0.6)$ & $\mathrm{I}(0.1)$ & - \\
\hline - with CAM & 0 & - & - & - & - & - & - \\
\hline Antiadrenergic agents & $17(1.0)$ & $4(0.6)$ & $13(1.2)$ & I (I.6) & $7(1.4)$ & $6(0.8)$ & $3(0.9)$ \\
\hline - with CAM & - & - & - & - & - & - & - \\
\hline Total & $1705(100)$ & $625(100)$ & $1080(100)$ & $63(100)$ & $517(100)$ & $796(100)$ & $329(100)$ \\
\hline
\end{tabular}

I Calcium channel blockers, ' complementary and alternative remedies

received other forms of antihypertensive monotherapy (i.e. angiotensin II receptor antagonists (11.7\%), alpha-1 blockers $(0.2 \%)$, and antiadrenergic agents $(1.0 \%))$.

With regard to combination drug treatments ( $\mathrm{n}=845$ prescriptions), the most commonly prescribed option was a diuretic plus an ACE inhibitor (31.2\% of all dual therapies), followed by a diuretic plus an angiotensin II receptor antagonist $(25.8 \%$ of all dual therapies). The latter option was prescribed significantly more often for women (29.1\% in women vs. $19.4 \%$ in men; $P=0.018$ ). Other dual and triple therapies ranged far behind. The most commonly prescribed fixed-dose coformulations were a diuretic plus an ACE inhibitor and a diuretic plus an angiotensin II receptor antagonist (table 3).

Prescription rates for patients with specific comorbidities were also compared to overall prescription rates. Patients with heart failure were more likely to be prescribed ACE inhibitors (32.8\% versus $24.5 \%$ for all patients) and diuretics $(23.4 \%$ versus $15.7 \%)$, and less likely to be prescribed beta-blockers (12.5\% versus $29.4 \%$ ) (table 4 ). Patients with renal insufficiency were also less likely to be prescribed beta-blockers $(15.7 \%)$, as were patients who had experienced a myocardial infarction $(18.3 \%)$; these patients were more likely, however, to be prescribed a diuretic $(21.3 \%$ in patients with renal insufficiency and $23.3 \%$ in patients after myocardial infarction).

With regard to combination treatments, patients with heart failure were more likely to be prescribed an ACE inhibitor plus a diuretic $(60 \%$ versus $31.3 \%$ for all patients), as were patients with renal insufficiency $(40.7 \%)$. Patients with diabetes were more likely to receive a diuretic plus an angiotensin II receptor antagonist than an ACE inhibitor plus a diuretic (28.4\% versus $25.1 \%$; see table 5 ).

\section{Factors associated with specific hypertensive agents}

Table 6 shows the adjusted OR for factors associated with specific antihypertensive agents independent of treatment regimen. Patient gender, age, and comorbidities had an impact on which treatment was prescribed. Men were more likely to receive an ACE inhibitor $(\mathrm{OR}=1.44 ; 95 \%$ CI 1.20-1.74) or an angiotensin II receptor antagonist $(\mathrm{OR}=1.62 ; 95 \%$ CI $1.26-2.09)$, but were less likely to receive a CAM remedy $(\mathrm{OR}=0.66 ; 95 \%$ CI $0.54-0.80)$. Patients older than 60 were more likely to be prescribed a CCB $(\mathrm{OR}=1.70 ; 95 \%$ CI 1.34-2.14), whereas patients under 60 were more likely to be prescribed a beta-blocker 
Table 3: Prescription of combination therapy according to age group and gender

\begin{tabular}{|c|c|c|c|c|c|c|c|}
\hline \multirow[t]{2}{*}{ Antihypertensives } & \multirow[t]{2}{*}{ Total } & \multicolumn{2}{|c|}{ Gender } & \multicolumn{4}{|c|}{ Age group [years] } \\
\hline & & Male & Female & Under 40 & $40-59$ & $60-79$ & 80 or older \\
\hline & [N (\%)] & [n (\%)] & [n (\%)] & [n (\%)] & [n (\%)] & [n (\%)] & [n (\%)] \\
\hline 2-drug combinations & $845(100)$ & $288(100)$ & $557(100)$ & $29(100)$ & $192(100)$ & $470(100)$ & $154(100)$ \\
\hline \multicolumn{8}{|l|}{ Diuretic plus } \\
\hline \multicolumn{8}{|l|}{ Beta-blocker } \\
\hline - Fixed-dose coformulation & $67(7.9)$ & $12(4.1)$ & $55(9.9)$ & $5(17.2)$ & $19(9.9)$ & $33(7.0)$ & $10(6.5)$ \\
\hline - Monopreparation & $53(6.3)$ & $15(5.2)$ & $38(6.8)$ & I (3.4) & $19(9.9)$ & $24(5.1)$ & $9(5.8)$ \\
\hline \multicolumn{8}{|l|}{$C C B^{\prime}$} \\
\hline - Fixed-dose coformulation & $\mathrm{I}(0.1)$ & $\mathrm{I}(0.3)$ & - & - & - & $\mathrm{I}(0.2)$ & - \\
\hline - Monopreparation & $30(3.6)$ & $11(3.8)$ & $19(3.4)$ & - & $3(1.6)$ & $15(3.2)$ & $12(7.8)$ \\
\hline \multicolumn{8}{|l|}{ ACE inhibitor } \\
\hline - Fixed-dose coformulation & $208(24.6)$ & $72(25.0)$ & $136(24.4)$ & I (3.4) & $4 \mid(2 \mid .4)$ & $128(27.2)$ & $38(24.7)$ \\
\hline - Monopreparation & $56(6.6)$ & $23(8.0)$ & $33(5.9)$ & - & $9(4.7)$ & $32(6.8)$ & $15(9.7)$ \\
\hline \multicolumn{8}{|l|}{ Angiotensin II antagonist } \\
\hline - Fixed-dose coformulation & $207(24.5)$ & $55(19.1)$ & $152(27.3)$ & $2(6.9)$ & $45(23.4)$ & $126(26.8)$ & $34(22.1)$ \\
\hline - Monopreparation & $11(1.3)$ & I $(0.3)$ & $10(1.8)$ & - & $2(1.0)$ & $5(1.1)$ & $4(2.6)$ \\
\hline \multicolumn{8}{|l|}{ CCBs plus } \\
\hline \multicolumn{8}{|l|}{ Beta-blocker } \\
\hline - Fixed-dose coformulation & - & & - & - & - & - & - \\
\hline - Monopreparation & $38(4.5)$ & $13(4.5)$ & $25(4.5)$ & $10(34.5)$ & $4(2.1)$ & $20(4.3)$ & $4(2.6)$ \\
\hline \multicolumn{8}{|l|}{ ACE inhibitor } \\
\hline - Fixed-dose coformulation & $\mathrm{I}(0.1)$ & $\mathrm{I}(0.3)$ & - & - & - & I (0.2) & - \\
\hline - Monopreparation & $30(3.6)$ & $11(3.8)$ & $19(3.4)$ & I (3.4) & $9(4.7)$ & $16(3.4)$ & $4(2.6)$ \\
\hline \multicolumn{8}{|l|}{ Angiotensin II antagonist } \\
\hline - Fixed-dose coformulation & - & - & - & - & - & - & - \\
\hline - Monopreparation & $20(2.4)$ & $9(3.1)$ & II (2.0) & - & $4(2.1)$ & $14(3.0)$ & $2(1.3)$ \\
\hline \multicolumn{8}{|l|}{ Beta-blockers plus } \\
\hline \multicolumn{8}{|l|}{ Other antihypertensive } \\
\hline - Fixed-dose coformulation & $5(0.6)$ & - & $5(0.9)$ & - & - & $4(0.9)$ & I (6.0) \\
\hline - Monopreparation & $81(9.6)$ & $52(18.1)$ & $29(5.2)$ & $7(24.1)$ & $33(17.2)$ & $33(7.0)$ & $8(5.2)$ \\
\hline Other & $37(4.4)$ & $12(4.2)$ & $25(4.5)$ & $2(6.9)$ & $4(2.1)$ & $18(3.8)$ & $13(8.4)$ \\
\hline 3-drug combinations & $104(100)$ & $58(100)$ & $46(100)$ & $3(100)$ & $31(100)$ & $58(100)$ & $12(10)$ \\
\hline \multicolumn{8}{|c|}{ Diuretic plus $A C E$ inhibitor plus $C C B$} \\
\hline \multicolumn{8}{|l|}{ - Monopreparation } \\
\hline & $2(1.9)$ & $2(3.4)$ & - & - & - & I ( 1.7$)$ & I (8.3) \\
\hline \multicolumn{8}{|l|}{ Diuretic plus beta-blocker plus ${ }^{2}$} \\
\hline - Monopreparation & $57(54.8)$ & $27(46.6)$ & $30(65.2)$ & I (33.3) & $20(64.5)$ & $30(51.7)$ & $6(50.0)$ \\
\hline \multicolumn{8}{|c|}{ Diuretic plus antiadrenergic agents plus ${ }^{2}$} \\
\hline \multicolumn{8}{|l|}{ - Monopreparation } \\
\hline & $6(5.8)$ & $5(8.6)$ & $\mathrm{I}(2.2)$ & - & $4(12.9)$ & $2(3.4)$ & - \\
\hline Other & $39(37.5)$ & $24(4 \mid .4)$ & $15(32.6)$ & $2(66.7)$ & $7(22.6)$ & $25(43.1)$ & $5(41.7)$ \\
\hline
\end{tabular}


Table 4: Prescription of monotherapies for comorbidities

\begin{tabular}{|c|c|c|c|c|c|c|c|c|c|c|c|}
\hline \multirow[t]{2}{*}{ Antihypertensives } & \multirow[t]{2}{*}{ Total } & \multirow{2}{*}{$\begin{array}{l}\text { Without } \\
\text { comorbidities }\end{array}$} & \multicolumn{9}{|c|}{ Comorbidities $^{3}$} \\
\hline & & & $\begin{array}{l}\text { Diabetes } \\
\text { mellitus }\end{array}$ & $\begin{array}{c}\text { Renal } \\
\text { insufficiency }\end{array}$ & $\begin{array}{l}\text { Hypercholes } \\
\text { terolaemia }\end{array}$ & CHD & $\begin{array}{c}\text { Post } \\
\text { myocardial } \\
\text { infarction }\end{array}$ & $\begin{array}{l}\text { Heart } \\
\text { failure }\end{array}$ & Stroke & Obesity & $\begin{array}{c}\text { Asthmal } \\
\text { COPD }\end{array}$ \\
\hline & [N (\%)] & [n (\%)] & [n (\%)] & [n (\%)] & [n (\%)] & [n (\%)] & [n (\%)] & [n (\%)] & [n (\%)] & [n (\%)] & [n (\%)] \\
\hline $\mathrm{CCB}^{\prime}$ & $527(17.1)$ & $88(18.6)$ & $55(15.9)$ & $26(20.5)$ & 97 (I5.4) & $58(14.5)$ & $12(20.0)$ & $12(18.8)$ & $56(20.4)$ & $47(13.6)$ & $76(20.8)$ \\
\hline - with CAM² & 14 & 5 & 2 & - & 1 & 1 & - & - & 2 & - & 3 \\
\hline Diuretic & $514(16.7)$ & 77 (16.2) & $46(13.3)$ & $27(21.3)$ & $58(9.2)$ & $73(18.3)$ & $14(23.3)$ & $15(23.4)$ & $54(19.6)$ & $63(18.2)$ & $87(23.8)$ \\
\hline - with CAM & 41 & 19 & 2 & I & 4 & 3 & - & - & 2 & 5 & 5 \\
\hline Beta-blocker & $882(28.7)$ & 151 (31.9) & $113(32.8)$ & $20(15.7)$ & $225(35.7)$ & II $4(28.5)$ & II (I8.3) & $8(12.5)$ & $61(22.2)$ & $96(27.7)$ & $83(22.7)$ \\
\hline - with CAM & 36 & 13 & 3 & - & 8 & 3 & - & - & 4 & 2 & 3 \\
\hline ACE inhibitor & $729(23.6)$ & $94(19.8)$ & $75(21.7)$ & $37(29.1)$ & $147(23.3)$ & $113(28.3)$ & $17(28.3)$ & $21(32.8)$ & $79(28.7)$ & $85(24.6)$ & $61(16.7)$ \\
\hline - with CAM & 33 & 6 & 4 & 3 & 6 & 9 & - & - & 2 & 2 & I \\
\hline $\begin{array}{l}\text { Angiotensin II } \\
\text { antagonist }\end{array}$ & $363(11.8)$ & $64(13.5)$ & $50(14.5)$ & II (8.7) & $86(13.6)$ & $38(9.5)$ & $5(8.3)$ & 7 (10.9) & $18(6.5)$ & 48 (I3.9) & $36(9.8)$ \\
\hline - with CAM & 23 & 8 & 1 & 1 & 4 & 1 & - & - & I & 4 & 3 \\
\hline Alpha-I blocker & $20(0.7)$ & - & $\mathrm{I}(0.3)$ & $\mathrm{I}(0.8)$ & $4(0.6)$ & $\mathrm{I}(0.3)$ & I (I.7) & - & $3(1.1)$ & $3(0.9)$ & $6(1.6)$ \\
\hline - with CAM & - & - & - & - & - & - & - & - & - & - & - \\
\hline Antiadrenergic agent & $44(1.4)$ & - & $5(1.4)$ & $5(3.9)$ & $14(2.2)$ & $3(0.8)$ & - & I (I.6) & $4(1.5)$ & $4(1.2)$ & $8(2.2)$ \\
\hline - with CAM & I & - & - & - & - & - & - & - & - & - & 1 \\
\hline Total & $3088(100)$ & $474(100)$ & $345(100)$ & $127(100)$ & $631(100)$ & $400(100)$ & $60(100)$ & $64(100)$ & $275(100)$ & $346(100)$ & $366(100)$ \\
\hline
\end{tabular}

I Calcium channel blocker, ${ }^{2}$ complementary remedy, ${ }^{3}$ double entries possible 
Table 5: Prescription of combination therapy for comorbidities

\begin{tabular}{|c|c|c|c|c|c|c|c|c|c|c|c|}
\hline \multirow[t]{2}{*}{ Antihypertensives } & \multirow[t]{2}{*}{ Total } & \multirow{2}{*}{$\begin{array}{r}\text { Without } \\
\text { comorbidities }\end{array}$} & \multicolumn{9}{|c|}{ Comorbidities $^{3}$} \\
\hline & & & $\begin{array}{l}\text { Diabetes } \\
\text { mellitus }\end{array}$ & $\begin{array}{c}\text { Renal } \\
\text { insufficiency }\end{array}$ & $\begin{array}{l}\text { Hypercholes } \\
\text { terolaemia }\end{array}$ & CHD & $\begin{array}{c}\text { Post } \\
\text { myocardial } \\
\text { infarction }\end{array}$ & $\begin{array}{l}\text { Heart } \\
\text { failure }\end{array}$ & Stroke & Obesity & $\begin{array}{c}\text { Asthma/ } \\
\text { COPD }\end{array}$ \\
\hline & {$[N(\%)]$} & [n (\%)] & [n (\%)] & [n (\%)] & [n (\%)] & [n (\%)] & [n (\%)] & [n (\%)] & [n (\%)] & [n (\%)] & [n (\%)] \\
\hline 2-drug & $1613(100)$ & $186(100)$ & $215(100)$ & $86(100)$ & $358(100)$ & $247(100)$ & $36(100)$ & $30(100)$ & $139(100)$ & $218(100)$ & $98(100)$ \\
\hline
\end{tabular}

combinations

Diuretic plus

Beta-blocker

Fixed-dose

coformulation

$103(6.4) \quad 17(9.2)$

II (5.I)

$2(2.3)$

$33(9.2)$

$14(5.7)$

$-$

I (3.3)

$4(2.9)$

$20(9.2)$

I (I.0)

Monopreparation

$106(6.6)$

14 (7.5)

$12(5.6)$

$5(5.8)$

$20(5.6)$

$15(6.1)$

$2(5.6)$

$9(6.5)$

$16(7.3)$

$3(13.3)$

CCB'

Fixed-dose

coformulation

$2(0.1)$

Monopreparation

58 (3.6)

7 (3.8)

$2(0.9)$

$6(7.0)$

$8(2.2)$

9 (3.6)

$2(5.6)$

$3(10.0)$

$5(3.2)$

ACE inhibitor

Fixed-dose

$372(23.1) \quad 43(23.1)$

$54(25.1)$

$28(32.6)$

75 (20.9)

$52(21.1)$

5 (13.9)

$16(53.3)$

$36(26.1)$

7 (8.1)

$24(6.7)$

$26(10.5)$

5 (13.9)

2 (6.7)

II (8.0)

(22.9)

Angiotensin II

antagonist

Fixed-dose

$132(8.2)$

$4(2.2) \quad 15(7.0)$

96 (24.6) $52(28.0)$

6I (28.4)

91 (25.4)

$4(11.1)$

5 (16.7)

3 I (22.5)

$29(29.6)$

Monopreparation

23 (1.4)

2 (I.I)

$2(0.9) \quad 2(2.3)$

7 (2.0)

4 (1.6)

- I (3.3)

$3(2.2)$

I (0.5)

I (I.0)

CCB plus

\section{Beta-blocker}

Fixed-dose

coformulation

Monopreparation

77 (4.8)

$15(8.1)$

$12(5.6)$

$5(5.8)$

II (3.I)

II (4.5)

4 (II.I)

$8(5.8)$

7 (3.2)

$4(4.1)$

ACE inhibitor 
Table 5: Prescription of combination therapy for comorbidities (Continued)

\begin{tabular}{|c|c|c|c|c|c|c|c|c|c|c|c|}
\hline $\begin{array}{l}\text { Fixed-dose } \\
\text { coformulation }\end{array}$ & I (0.I) & I (0.5) & - & - & - & - & - & - & - & - & - \\
\hline Monopreparation & $65(4.0)$ & $7(3.8)$ & $8(3.7)$ & $6(7.0)$ & $13(3.6)$ & $13(5.3)$ & $3(8.3)$ & - & $4(2.9)$ & $4(1.8)$ & $7(7.1)$ \\
\hline \multicolumn{12}{|l|}{$\begin{array}{l}\text { Angiotensin II } \\
\text { antagonist }\end{array}$} \\
\hline $\begin{array}{l}\text { Fixed-dose } \\
\text { coformulation }\end{array}$ & - & - & - & - & - & - & - & - & - & - & - \\
\hline Monopreparation & $35(2.2)$ & $7(3.8)$ & $2(0.9)$ & - & $8(2.2)$ & $6(2.4)$ & I (2.8) & - & $5(3.6)$ & $5(2.3)$ & I (I.0) \\
\hline
\end{tabular}

\section{Beta-blocker plus}

other

antihypertensive

Fixed-dose

$(0.6)$

coformulation

9 (0.6) $\quad \mathrm{I}(0.5)$

(0.5)

$172(10.7)$

$13(7.0)$

$26(12.1)$

- $\quad 4(1.1)$

I (0.4)

$\begin{array}{lll}- & \mathrm{I}(3.3) \quad 2(1.3)\end{array}$

Other

3-drug

$3(1.6)$

$10(4.7)$

$47(13.1) \quad 37(14.9)$

I (3.3)

$13(9.4) \quad 19(8.7)$

$2(2.0)$

$222(100) \quad 15(100)$

$10(4.7)$

$6(7.0)$

$17(4.7) \quad 9(3.6)$

-

-

Diuretic plus ACE

inhibitor plus CCB

Monopreparation

8 (3.6)

I (2.4)

I (5.9)

$2(3.8)$

$2(6.3)$

$-$

Diuretic plus beta-

blocker plus 2

Monopreparation

I 20 (54. I)

$6(40.0)$

$26(61.9)$

5 (29.4)

$26(50.0)$

$19(59.4)$

I (50.0)

$9(60.0)$

$17(68.0)$

II (52.4)

Diuretic plus

antiadrenergic agent

$\begin{array}{ll}\stackrel{0}{0} & \text { plus }^{2} \\ \stackrel{0}{0} & \text { Monopreparation }\end{array}$

$10(4.5)$

$10(4.5)$

2 (II.8)

$3(5.8)$

I (3.I)

-

$-$

$-$

$2(9.5)$

Other

84 (37.8) $7(46.7)$

$15(35.7) \quad 9(52.9)$

$21(40.4) \quad 10(31.3)$

I (50.0)

I (100)

$6(40.0)$

$\begin{array}{cc}- & 2(9.5) \\ 8(32.0) & 6(28.6)\end{array}$

I Calcium channel blocker, ${ }^{2} \mathrm{CCB}, \mathrm{ACE}$ inhibitor, angiotensin II antagonist, alpha-blocker, or dihydrazine, ${ }^{3}$ double entries possible 
Table 6: Multiple logistic regression: Factors associated with special hypertensive medication

\begin{tabular}{|c|c|c|c|c|c|c|}
\hline & CCBs & Diuretics & Beta-blockers & ACE inhibitors & Angiotensin II antagonists & CAM remedy \\
\hline Factor & \multicolumn{6}{|c|}{ Adjusted OR (95\% Cl) } \\
\hline Gender (male) & $\begin{array}{l}1.21 \\
(0.99-1.48)\end{array}$ & $\begin{array}{l}1.02 \\
(0.83-1.25)\end{array}$ & $\begin{array}{l}1.15 \\
(0.96-1.36)\end{array}$ & $\begin{array}{l}1.44 \\
(1.20-1.74)^{*}\end{array}$ & $\begin{array}{l}1.62 \\
(1.26-2.09)^{*}\end{array}$ & $\begin{array}{l}0.66 \\
(0.54-0.80)^{*}\end{array}$ \\
\hline $\begin{array}{l}\text { Age } \\
\text { ( } 60 \text { years or older) }\end{array}$ & $\begin{array}{l}1.70 \\
(1.34-2.14)^{*}\end{array}$ & $\begin{array}{l}1.10 \\
(0.88-1.38)\end{array}$ & $\begin{array}{l}0.50 \\
(0.41-0.60)^{*}\end{array}$ & $\begin{array}{l}0.87 \\
(0.70-1.06)\end{array}$ & $\begin{array}{l}0.75 \\
(0.57-0.98)^{*}\end{array}$ & $\begin{array}{l}1.16 \\
(0.95-1.42)\end{array}$ \\
\hline Comorbidies ${ }^{\prime}$ & $\begin{array}{l}0.87 \\
(0.64-1.18)\end{array}$ & $\begin{array}{l}1.09 \\
(0.79-1.49)\end{array}$ & $\begin{array}{l}0.99 \\
(0.76-1.30)\end{array}$ & $\begin{array}{l}1.57 \\
(1.16-2.11)^{*}\end{array}$ & $\begin{array}{l}0.46 \\
(0.30-0.69)^{*}\end{array}$ & $\begin{array}{l}0.91 \\
(0.69-1.21)\end{array}$ \\
\hline Diabetes & $\begin{array}{l}1.01 \\
(0.79-1.29)\end{array}$ & $\begin{array}{l}0.98 \\
(0.76-1.25)\end{array}$ & $\begin{array}{l}1.63 \\
(1.33-2.00)^{*}\end{array}$ & $\begin{array}{l}0.85 \\
(0.67-1.07)\end{array}$ & $\begin{array}{l}1.92 \\
(1.42-2.60)^{*}\end{array}$ & $\begin{array}{l}0.55 \\
(0.42-0.73)^{*}\end{array}$ \\
\hline Renal insufficiency & $\begin{array}{l}1.49 \\
(1.09-2.05)^{*}\end{array}$ & $\begin{array}{l}1.33 \\
(0.96-1.83)\end{array}$ & $\begin{array}{l}0.58 \\
(0.4 I-0.8 I)^{*}\end{array}$ & $\begin{array}{l}1.05 \\
(0.76-1.44)\end{array}$ & $\begin{array}{l}0.66 \\
(0.40-1.10)\end{array}$ & $\begin{array}{l}0.89 \\
(0.62-1.28)\end{array}$ \\
\hline Hypercholesterolaemia & $\begin{array}{l}1.09 \\
(0.87-1.37)\end{array}$ & $\begin{array}{l}0.70 \\
(0.56-0.88)^{*}\end{array}$ & $\begin{array}{l}1.29 \\
(1.06-1.56)^{*}\end{array}$ & $\begin{array}{l}0.93 \\
(0.76-1.15)\end{array}$ & $\begin{array}{l}1.74 \\
(1.28-2.36)^{*}\end{array}$ & $\begin{array}{l}0.59 \\
(0.47-0.75)^{*}\end{array}$ \\
\hline CHD & $\begin{array}{l}1.00 \\
(0.79-1.26)\end{array}$ & $\begin{array}{l}1.32 \\
(1.05-1.66)^{*}\end{array}$ & $\begin{array}{l}1.68 \\
(1.04-1.57)^{*}\end{array}$ & $\begin{array}{l}1.31 \\
(1.05-1.62)^{*}\end{array}$ & $\begin{array}{l}1.12 \\
(0.82-1.53)\end{array}$ & $\begin{array}{l}0.82 \\
(0.64-1.05)\end{array}$ \\
\hline Post myocardial infarction & $\begin{array}{l}1.27 \\
(0.78-2.07)\end{array}$ & $\begin{array}{l}1.43 \\
(0.89-2.32)\end{array}$ & $\begin{array}{l}1.30 \\
(0.83-2.04)\end{array}$ & $\begin{array}{l}1.69 \\
(1.09-2.62)^{*}\end{array}$ & $\begin{array}{l}0.94 \\
(0.47-1.89)\end{array}$ & $\begin{array}{l}0.37 \\
(0.17-0.8 I)^{*}\end{array}$ \\
\hline Heart failure & $\begin{array}{l}0.72 \\
(0.42-1.23)\end{array}$ & $\begin{array}{l}0.98 \\
(0.59-1.60)\end{array}$ & $\begin{array}{l}0.30 \\
(0.16-0.59)^{*}\end{array}$ & $\begin{array}{l}1.03 \\
(0.64-1.64)\end{array}$ & $\begin{array}{l}0.93 \\
(0.44-1.97)\end{array}$ & $\begin{array}{l}0.84 \\
(0.51-1.38)\end{array}$ \\
\hline Stroke & $\begin{array}{l}1.22 \\
(0.93-1.50)\end{array}$ & $\begin{array}{l}1.18 \\
(0.90-1.56)\end{array}$ & $\begin{array}{l}1.02 \\
(0.79-1.31)\end{array}$ & $\begin{array}{l}1.22 \\
(0.94-1.58)\end{array}$ & $\begin{array}{l}1.29 \\
(0.87-1.89)\end{array}$ & $\begin{array}{l}0.54 \\
(0.40-0.74)^{*}\end{array}$ \\
\hline Obesity & $\begin{array}{l}0.98 \\
(0.76-1.28)\end{array}$ & $\begin{array}{l}1.49 \\
(1.16-1.01)^{*}\end{array}$ & $\begin{array}{l}0.79 \\
(0.64-0.99)^{*}\end{array}$ & $\begin{array}{l}1.11 \\
(0.88-1.40)\end{array}$ & $\begin{array}{l}1.07 \\
(0.77-1.48)\end{array}$ & $\begin{array}{l}0.74 \\
(0.57-0.97)^{*}\end{array}$ \\
\hline Asthma/COPD & $\begin{array}{l}1.4 \mathrm{I} \\
(1.06-1.88)^{*}\end{array}$ & $\begin{array}{l}1.69 \\
(1.23-2.23)^{*}\end{array}$ & $\begin{array}{l}0.94 \\
(0.7 \mid-1.23)\end{array}$ & $\begin{array}{l}0.73 \\
(0.54-0.98)^{*}\end{array}$ & $\begin{array}{l}1.44 \\
(0.97-2.13)\end{array}$ & $\begin{array}{l}0.91 \\
(0.67-1.24)\end{array}$ \\
\hline
\end{tabular}

I at least one of the following indications in table 6

* OR significantly different from I

$(\mathrm{OR}=0.50 ; 95 \% \mathrm{CI} 0.41-0.60)$. With regard to specific comorbidities, patients with diabetes, for example, were more likely to be prescribed a beta-blocker $(\mathrm{OR}=1.63$; 95\% CI 1.33-2.00) or an angiotensin II receptor antagonist $(\mathrm{OR}=1.92 ; 95 \%$ CI 1.42-2.60). Concomitant diseases, such as diabetes mellitus $(\mathrm{OR}=0.55 ; 95 \% \mathrm{CI} 0.42$ $0.73)$, hypercholesterolaemia $(\mathrm{OR}=0.59 ; 95 \%$ CI 0.47 0.75 ), obesity (OR $=0.74 ; 95 \%$ CI 0.57-0.97), stroke (OR $=0.54 ; 95 \%$ CI $0.40-0.74)$, and post myocardial infarction $(\mathrm{OR}=0.37 ; 95 \% \mathrm{CI} 0.17-0.81)$, reduced the odds of being prescribed a CAM remedy.

Power calculation was performed for the odds ratios from the logistic regression models in table 6 based on the algorithm provided in [35]. At a significance level of 0.05, the present study had $90 \%$ to $100 \%$ power to detect a change of 0.1 in the ORs (exceptions: post myocardial infarction: 0.42-0.68; heart failure 0.40-0.71).

\section{Discussion}

A number of studies have investigated physician adherence to hypertension guidelines by analysing patient medical records or physician survey data. Although surveys are a useful tool, most have methodological limitations that could cause physician adherence to be underestimated. The present study relies, instead, on electronic prescription records and thus is not subject to the reporting or retrieval biases inherent to paper-based surveys.

The majority of patients in the present study were treated with classic antihypertensive monotherapies. Of these agents, beta-blockers were prescribed most frequently, fol- 
lowed by ACE inhibitors, CCBs, and diuretics. Patient gender, age, and the presence of comorbidities had a significant influence on which treatment was prescribed.

\section{Limitations}

The present study has several important limitations. First, additional data on the hypertension diagnoses are lacking, such as blood pressure, smoking status, and lifestyle factors. Second, data on subsequent medication use in patients who switched physicians were unavailable, as were data on whether a patient was being treated for the first time with an antihypertensive agent. Third, from a statistical point of view, the subgroup analyses may be underpowered in cases where differences were small. Fourth, although our study population can be regarded as fairly representative of the overall population of German physicians specialized in anthroposophic treatment, it is not representative of all CAM physicians in Germany, who offer a range of further treatments, including those from the field of traditional Chinese medicine or integrative treatment programmes including Tai Chi $[36,37]$. Such treatments are not prescribed frequently, however. Finally, the present study lacks a direct comparison group. Further research on this subject would benefit from including a comparison group of CAM physicians, such as homoeopaths, or of conventional primary care physicians.

\section{Study population}

Of the 3672 eligible patients who received a prescription during the study, $35.9 \%(\mathrm{n}=1320)$ were prescribed conventional and/or CAM treatment for hypertension at least once. This is comparable to other studies $[3,4,38]$, in which a large proportion of patients have also been older (i.e. $65 \%$ aged 60 years or more) [39] and a higher CAM treatment rate was observed in women [40-43]. Although the types and rates of comorbidities in the present sample were generally similar to those observed in other surveys, the rates of diabetes and coronary heart disease experienced by our patients were slightly lower [44], which may be attributable to higher educational attainment or greater health awareness among users of complementary medicine [45].

\section{Prescription of antihypertensive treatments and adherence to guidelines}

Each hypertensive patient received an average of 2.5 prescriptions per year, which at first glance might seem indicative of undertreatment. Indeed, this interpretation would be in accordance with the results of the HYDRA trial, in which patients were not provided with adequate hypertensive treatment by their GPs [38]. Nevertheless, the low average number of prescriptions per year in the present study may be attributable, at least in part, to two of the study's above mentioned limitations: (1) the inclusion in the cohort of patients being treated for the first time with antihypertensive therapy, and (2) the lack of data on subsequent medication use in patients who switched physicians.

In addition to lifestyle changes, current guidelines recommend initiating antihypertensive treatment using one or more agents from the following five drug classes: (thiazide) diuretics, beta-blockers, CCBs, ACE inhibitors, and angiotensin receptor blockers. Other substances are recommended only as adjunctive treatment. In the present study, only $2 \%$ of patients had conventional prescriptions that did not include any agents from these five classes. Monotherapies accounted for $64 \%$ of all prescribed treatments, and the proportion of patients receiving monotherapy was high compared to the German MONICA Study (50\%) and similar to that observed in Israel (64\%) and Finland (59\%) [46]. In accordance with other studies, beta-blockers were prescribed most often, followed by ACE inhibitors and the combination of a diuretic plus an ACE inhibitor $[39,46]$. When available, fixed-dose coformulations were used frequently, accounting for $55.4 \%$ of all two-drug combinations; this is likely attributable to the desire to increase patient compliance.

The finding that there are significant differences in the prescription rates for various antihypertensive agents according to age, gender, and comorbidities is in accordance with the results reported by Pears et al. [47].

After being adjusted for comorbidity and age, the results of the present study show that women were less likely to receive ACE inhibitors or angiotensin II receptor antagonists $[48,49]$. Beta-blockers were prescribed primarily for patients under the age of 60 . These findings concur with those in comparable national and international surveys of antihypertensive drug use in primary care [38].

In 2003 Pittrow et al. showed that in German primary care, hypertensive patients with comorbidities do not receive the individualized treatment recommended in the current Germany Hypertension Society guidelines. For example, although CCBs and beta-blockers are the preferred option in CHD patients with hypertension, ACE inhibitors and diuretics were prescribed most frequently [39]. In the present study, there was also a significantly higher prescription rate for diuretics $(\mathrm{OR}=1.32 ; 95 \% \mathrm{CI}$ $1.05-1.66)$ or ACE inhibitors $(\mathrm{OR}=1.31 ; 95 \%$ CI 1.051.62) in CHD patients; however, the OR for the use of beta-blockers, which are recommended in the German Hypertension Society guidelines, was even higher (1.68; 95\% CI 1.04-1.57). For patients with diabetes, the OR for receiving beta-blockers, which would also address the high cardiac risk associated with diabetes, was significantly greater than $1(\mathrm{OR}=1.63 ; 95 \%$ CI 1.33-2.00) [39]. 
The OR for patients with prior myocardial infarction was significantly greater than 1 for ACE inhibitors $(O R=1.69$; 95\% CI 1.09-2.62), which are also recommended in the guidelines, but there was not a significantly lower prescription rate for CCBs (OR $=1.27 ; 95 \%$ CI 0.78-2.07), which are not recommended.

The prescription rates for CCBs in patients with heart failure (12 out of 527 patient, or $2.3 \%$ ), beta-blockers in asthma/COPD patients ( 83 out of 882 patients, or $9.4 \%$ ), and alpha-1-blockers in patients with renal insufficiency (1 out of 20 patients, or 5.0\%) - all of which are contraindicated in the respective patient group - were below $10 \%$. After adjustment for other factors, multiple logistic regression did not result in significantly lower prescription rates for CCBs in patients with heart failure $(\mathrm{OR}=0.72 ; 95 \% \mathrm{CI}$ $0.42-1.23)$ or for beta-blockers in patients with asthma/ COPD $(\mathrm{OR}=0.94 ; 95 \%$ CI 0.71-1.23).

\section{CAM remedies}

Although CAM remedies are not recommended in any of the currently available guidelines, they were included in $19.1 \%$ of the prescriptions in the present study. This is not surprising considering that most patients who visit CAM physicians likely do so because they are seeking alternative treatments. It may be that this group of patients had only mild hypertension and received CAM remedies in addition to any conventional treatment and recommendations for lifestyle changes. The results of the present study strongly suggest that CAM remedies were prescribed mainly as adjuncts to antihypertensive monotherapy, most likely as a way to stimulate and harmonize the rhythmic system, assisting the body in regulating blood pressure with its own resources [50]. As expected, CAM medication was prescribed primarily to women and patients without comorbidities [40-43]. Altogether, the prescription rate for CAM remedies among patients with hypertension was very low compared to that among patients with other diseases, such as acute upper respiratory tract infections (79.8\%), otitis media, or dorsopathies, in the same network of physicians [51].

\section{Elderly patients with hypertension}

Due to methodological limitations, most earlier studies on hypertension have excluded patients older than 65 , thus failing to evaluate a subgroup of patients whose importance will only increase in the coming decades. The Hyvet study published in 2008 [52] was therefore an important addition to the literature, demonstrating the benefits of treating hypertension in patients aged 80 years or older. In accordance with current German Hypertension Society guidelines, the results of the present study show higher prescription rates for CCBs and diuretics in elderly patients, as well as an almost equal distribution of classic antihypertensive monotherapies among patients aged 80 years and older.

\section{Conclusions}

This paper is the first to provide insight into the hypertension treatment strategies of physicians specialized in CAM treatment using routine data from practice information systems. Despite the limitations of the available data, it can be concluded that the large majority of antihypertensive treatments prescribed by CAM physicians in the present study were compliant with the current guidelines of the German Hypertension Society. Deviations from these guidelines were seen in one of every seven patients receiving some form of CAM treatment.

\section{Competing interests}

The authors declare that they have no competing interests.

\section{Authors' contributions}

EJ helped design the study and acquire data, performed the statistical analysis, and drafted the manuscript. TO made substantial contributions to data interpretation and statistical analysis. $\mathrm{HCV}, \mathrm{AB}, \mathrm{MK}$, and $\mathrm{CMW}$ helped interpret the data and draft or critically revise the manuscript. HM conceived of the study and participated in its design and coordination. MK, CMW, SNW, and HM gave final approval of the version to be published. All authors read and approved the final manuscript.

\section{Acknowledgements}

The EvaMed network was supported by grants from the Software AG Foundation, Wala Heilmittel GmbH, and Weleda AG. The sponsors had no influence on the design or implementation of the study; the collection, management, or analysis of data; or the preparation, review, or approval of the manuscript. We would also like to thank Matthew D. Gaskins for copy-editing the text. Finally, we would like to express our special gratitude to all physicians participating in the EvaMed Network.

\section{References}

I. Kearney PM, Whelton M, Reynolds K, Muntner P, Whelton PK, He J: Global burden of hypertension: analysis of worldwide data. Lancet 2005, 365(9455):217-223.

2. Prugger C, Heuschmann PU, Keil U: [Epidemiology of hypertension in Germany and worldwide]. Herz 2006, 3 I (4):287-293.

3. Thamm M: [Blood pressure in Germany--current status and trends]. Gesundheitswesen 1999, 61:90-93.

4. Wolf-Maier K, Cooper RS, Banegas JR, Giampaoli S, Hense HW, Joffres M, Kastarinen M, Poulter N, Primatesta P, Rodriguez-Artalejo F, et al:: Hypertension prevalence and blood pressure levels in 6 European countries, Canada, and the United States. JAMA 2003, 289( I 8):2363-2369.

5. Zentralinstitut für die kassenärztliche Versorgung in der Bundesrepublik Deutschland. Ergebnisse: Panel-Basisstatistik [http://www.zi-berlin.de/morbilitaetsanalyse/downloads/ADTPanel-version3.pdf]

6. Anlauf M: Arzneiverordnungs-Report 2006. Aktuelle Daten, Kosten, Trends und Kommentare Edited by: Schwabe U, Paffrath D. Springer Verlag; 2006.

7. Schneider CA, Hagemeister J, Pfaff H, Mager G, Hopp HW: [Knowledge of guideline recommendations among internists and general practitioners regarding the diagnosis and treatment 
of arterial hypertension]. Z Arztl Fortbild Qualitatssich 200I, 95(5):339-344.

8. Harder S, Thurmann P, Thierolf C, Klepzig H: Prescription of cardiovascular drugs in outpatient care: a survey of outpatients in a German university hospital. Int J Clin Pharmacol Ther 1998, 36(4): 195-20I.

9. Fischer MA, Avorn J: Economic implications of evidence-based prescribing for hypertension: can better care cost less? JAMA 2004, 29I(15):1850-1856.

10. Vollmar HCRM, Popert U, Butzlaff M: [New Hypertension Guidelines - Need for Action among General Practitioners?] Zeitschrift für Allgemeinmedizin 2004, 80(6):237-242.

II. German Hypertension Society. Leitlinien zur Behandlung der arteriellen Hypertonie [http://www.paritaet.org/RR-Liga/]

12. Goldstein MK, Hoffman BB, Coleman RW, Tu SW, Shankar RD, O'Connor M, Martins S, Martins S, Advani A, Musen MA: Patient safety in guideline-based decision support for hypertension management: ATHENA DSS. Proc AMIA Symp 200I:2।4-2।8.

13. McKinstry B, Hanley J, Heaney D, McCloughan L, Elton R, Webb DJ: Impact on hypertension control of a patient-held guideline: a randomised controlled trial. $\mathrm{Br} J$ Gen Pract 2006, 56(532):842-847.

14. Wright $\mathrm{Cl}$, Van-Buren L, Kroner $\mathrm{Cl}$, Koning MM: Herbal medicines as diuretics: a review of the scientific evidence. J Ethnopharmacol 2007, I I 4(I): I-3I.

15. Steiner R, Wegmann I: Extending practical medicine: fundamental principles based on the science of the spirit. In GA 27 Bristol: Rudolf Steiner Press; 2000:I-144.

16. Chang WT, Dao J, Shao ZH: Hawthorn: potential roles in cardiovascular disease. Am J Chin Med 2005, 33(I): I-I0.

17. Schroder D, Weiser M, Klein P: Efficacy of a homeopathic Crataegus preparation compared with usual therapy for mild (NYHA II) cardiac insufficiency: results of an observational cohort study. Eur J Heart Fail 2003, 5(3):319-326.

18. Ghayur MN, Gilani AH: Ginger lowers blood pressure through blockade of voltage-dependent calcium channels. J Cardiovasc Pharmacol 2005, 45(I):74-80.

19. Cysarz D, Heckmann C, Kummell HC: [The effects of Cardiodoron on cardio-respiratory coordination--a literature review]. Forsch Komplementarmed Klass Naturheilkd 2002, 9(5):292-297.

20. Ernst E: Complementary/alternative medicine for hypertension: a mini-review. Wien Med Wochenschr 2005, I55(I7 18):386-39|

21. Amira OC, Okubadejo NU: Frequency of complementary and alternative medicine utilization in hypertensive patients attending an urban tertiary care centre in Nigeria. BMC Complement Altern Med 2007, 7:30.

22. Shafiq N, Gupta M, Kumari S, Pandhi P: Prevalence and pattern of use of complementary and alternative medicine (CAM) in hypertensive patients of a tertiary care center in India. Int Clin Pharmacol Ther 2003, 4 I(7):294-298.

23. Yeh GY, Davis RB, Phillips RS: Use of complementary therapies in patients with cardiovascular disease. Am J Cardiol 2006, 98(5):673-680.

24. Frenkel MA, Borkan JM: An approach for integrating complementary-alternative medicine into primary care. Fam Pract 2003, 20(3):324-332.

25. Cabana MD, Rand CS, Powe NR, Wu AW, Wilson MH, Abboud PA, Rubin HR: Why don't physicians follow clinical practice guidelines? A framework for improvement. JAMA 1999 , 282(I5): | 458- | 465 .

26. Freeborn DK, Shye D, Mullooly JP, Eraker S, Romeo J: Primary care physicians' use of lumbar spine imaging tests: effects of guidelines and practice pattern feedback. J Gen Intern Med 1997, I 2(10):619-625

27. Schers H, Wensing M, Huijsmans Z, van Tulder M, Grol R: Implementation barriers for general practice guidelines on low back pain a qualitative study. Spine (Phila Pa 1976) 200I, 26(I5):E348-353.

28. Espeland A, Baerheim A: Factors affecting general practitioners decisions about plain radiography for back pain: implications for classification of guideline barriers--a qualitative study. BMC Health Serv Res 2003, 3(I):8.
29. Matthes HTM, Jeschke E: Ein Pharmakovigilanzsystem für Arzneimittel der besonderen Therapierichtungen. EHK 2008:34-39.

30. Jeschke E, Ostermann T, Lüke C, Tabali M, Kröz M, Bockelbrink A Witt MC, Willich SN, Matthes H: Remedies Containing Asteraceae Extracts: A Prospective Observational Study of Prescribing Patterns and Adverse Drug Reactions in German Primary Care. Drug Saf 2009, 32(8): I- 16.

31. Jeschke E, Ostermann T, Tabali M, Bockelbrink A, Witt MC, Willich SN, Matthes H: Profiling Anthroposophic Medicine in Daily Practice - A Web-based, Prospective Multicentre Study. Forsch Komplementmed in press.

32. Arbeitsgruppe Erhebung und Nutzung von Sekundärdaten der Deutschen Gesellschaft für Sozialmedizin und Prävention; Arbeitsgruppe Epidemiologische Methoden der Deutschen Gesellschaft für Epidemiologie; Deutschen Gesellschaft für Medizinische Informatik; Biometrie und Epidemiologie; Deutschen Gesellschaft für Sozialmedizin und Prävention: [Good practice of secondary data analysis, first revision]. Gesundheitswesen 2008, 70(I):54-60

33. Jeschke E, Schad F, Pissarek J, Matthes B, Albrecht U, Matthes H [QuaDoSta - ein frei konfigurierbares System zur Unterstützung multizentrischer Datenerhebungen in medizinischer Versorgung und Forschung.]. GMS Med Inform Biom Epidemiol 2007, 3(2):Doc 10.

34. Kasenärztliche Bundesvereinigung: Grunddaten zur vertragsärztlichen Versorgung 2008 [http://www.zi-berlin.de/morbilita etsanalyse/downloads/ADT-Panel-version3.pdf]

35. Hsieh FY, Block DA, Larsen MD: A Simple Method of Sample Size Calculation for Linear and Logistic Regression. Statistics in Medicine 1998, 17:1623-1634.

36. Yeh GY, Wang C, Wayne PM, Phillips RS: The effect of tai chi exercise on blood pressure: a systematic review. Prev Cardiol 2008, II (2):82-89.

37. Li H, Liu LT: [Considerations about treatment programs of elderly patients with hypertension]. Zhong $X_{i} Y_{i}$ Jie He Xue Bao 2009, 7(7):607-610.

38. Sharma AM, Wittchen HU, Kirch W, Pittrow D, Ritz E, Goke B, Lehnert $\mathrm{H}$, Tschope $\mathrm{D}$, Krause $\mathrm{P}$, Hofler M, et al.: High prevalence and poor control of hypertension in primary care: cross-sectional study. J Hypertens 2004, 22(3):479-486.

39. Pittrow D, Kirch W, Bramlage P, Lehnert H, Hofler M, Unger T, Sharma AM, Wittchen HU: Patterns of antihypertensive drug utilization in primary care. Eur J Clin Pharmacol 2004, 60(2): $135-142$.

40. Fleming S, Rabago DP, Mundt MP, Fleming MF: CAM therapies among primary care patients using opioid therapy for chronic pain. BMC Complement Altern Med 2007, 7:15.

4l. Lawsin C, DuHamel K, Itzkowitz SH, Brown K, Lim H, Thelemaque L, Jandorf L: Demographic, medical, and psychosocial correlates to CAM use among survivors of colorectal cancer. Support Care Cancer 2007, I5(5):557-564.

42. Shinto L, Yadav V, Morris C, Lapidus JA, Senders A, Bourdette D: Demographic and health-related factors associated with complementary and alternative medicine (CAM) use in multiple sclerosis. Mult Scler 2006, I 2(I):94-100

43. Bishop FL Lewith GT: Who Uses CAM? A Narrative Review of Demographic Characteristics and Health Factors Associated with CAM Use. Evid Based Complement Alternat Med 2008 in press.

44. Borzecki AM, Wong AT, Hickey EC, Ash AS, Berlowitz DR: Identifying hypertension-related comorbidities from administrative data: what's the optimal approach? Am J Med Qual 2004, 19(5):201-206

45. Furnham A, Forey J: The attitudes, behaviors and beliefs of patients of conventional vs. complementary (alternative) medicine. J Clin Psychol 1994, 50(3):458-469.

46. Gasse C, Stieber J, Doring A, Keil U, Hense HW: Population trends in antihypertensive drug use: results from the MONICA Augsburg Project 1984 to 1995. J Clin Epidemiol 1999, 52(7):695-703.

47. Pears E, Hannaford PC, Taylor MW: Gender, age and deprivation differences in the primary care management of hypertension in Scotland: a cross-sectional database study. Fam Pract 2003, 20(1):22-31. 
48. Gasse C, Hense HW, Stieber J, Doring A, Liese AD, Heller G, Keil U: Factors associated with differences in antihypertensive drug treatment: results from the MONICA Augsburg Population Surveys 1989/90 and 1994/95. Soz Praventivmed 2002, 47(2): $128-142$.

49. Gu Q, Burt VL, Paulose-Ram R, Dillon CF: Gender differences in hypertension treatment, drug utilization patterns, and blood pressure control among US adults with hypertension: data from the National Health and Nutrition Examination Survey 1999-2004. Am J Hypertens 2008, 2 I(7):789-798.

50. Hildebrandt G: Reactive modifications of the autonomous time structure in the human organism. J Physiol Pharmacol I99I, 42(I):5-27.

5I. Jeschke E, Luke C, Ostermann T, Tabali M, Hubner J, Matthes H: [Prescribing practices in the treatment of upper respiratory tract infections in anthroposophic medicine]. Forsch Komplementmed 2007, 14(4):207-215.

52. Beckett NS, Peters R, Fletcher AE, Staessen JA, Liu L, Dumitrascu D, Stoyanovsky V, Antikainen RL, Nikitin Y, Anderson C, et al:: Treatment of hypertension in patients 80 years of age or older. $N$ Engl J Med 2008, 358(18): | 887-1898.

\section{Pre-publication history}

The pre-publication history for this paper can be accessed here:

http://www.biomedcentral.com/1471-2296/10/78/pre pub

Publish with Bio Med Central and every scientist can read your work free of charge

"BioMed Central will be the most significant development for disseminating the results of biomedical research in our lifetime. "

Sir Paul Nurse, Cancer Research UK

Your research papers will be:

- available free of charge to the entire biomedical community

- peer reviewed and published immediately upon acceptance

- cited in PubMed and archived on PubMed Central

- yours - you keep the copyright 